POS $\quad$ PROCEEDINGS

\title{
QCD resummations for boosted top production
}

\author{
Andrea Ferroglia \\ New York City College of Technology, 300 Jay Street \\ Brooklyn, NY 11201, USA \\ The Graduate School and University Center, The City University of New York \\ New York, NY 10016 USA \\ E-mail: aferrogliaecitytech.cuny.edu
}

Benjamin D. Pecjak*

Institute for Particle Physics Phenomenology, University of Durham

DH1 3LE Durham, UK

E-mail: ben.pecjak@durham.ac.uk

\section{Darren J. Scott}

Institute for Particle Physics Phenomenology, University of Durham

DH1 3LE Durham, UK

E-mail: d.j.scott@durham.ac.uk

\section{Li Lin Yang}

School of Physics and State Key Laboratory of Nuclear Physics and Technology

Peking University, Beijing 100871, China

E-mail: yanglilin@pku.edu.cn

\begin{abstract}
We present new results for QCD corrections to the top-pair invariant mass and top-quark $p_{T}$ distributions in boosted top-quark pair production at hadron colliders. They are derived from a formalism which allows the joint resummation of soft and small-mass logarithms at NNLL' order, thus taking into account all potentially large corrections in the boosted regime, where the partonic center-of-mass energy is parameterically much larger than the mass of the top quark. We match these results with those from standard soft-gluon resummation away from the small-mass limit to NNLL order and also with NLO fixed-order calculations, so that our results are valid in the maximum possible range of phase space. The resummation effects on the $p_{T}$ and top-pair invariant mass distributions are significant, bringing theory predictions into better agreement with experimental data compared to pure NLO calculations.
\end{abstract}

8th International Workshop on Top Quark Physics, TOP2015

14-18 September, 2015

Ischia, Italy

${ }^{*}$ Speaker. 


\section{Introduction}

Detailed studies of top-quark properties are interesting for a number of reasons (see [1] for a recent review). Many of these are related to the large value of the top-quark mass, $m_{t} \approx 173 \mathrm{GeV}$. For these reasons, top-quark pair production is a benchmark process at hadron colliders such as the LHC, and much work has gone into precision Standard Model calculations to match the evergrowing accuracy of experimental measurements.

Differential pair-production cross sections are largest in the region of phase space characterized by $\hat{s} \gtrsim 4 m_{t}^{2}$, with $\hat{s}$ the partonic center-of-mass energy squared. In such regions of phase space, QCD corrections can be calculated straightforwardly as a fixed-order expansion in the strong coupling constant $\alpha_{s}$. Such fixed-order calculations have now been carried out to high precision: results at next-to-next-to-leading order (NNLO) for differential top-pair production cross sections were presented at this conference and published recently in [2]. This tremendously impressive calculation will form the baseline description of top-pair production cross sections in perturbative QCD for years to come, and adds to previous results for the total cross section [3] and the forward-backward asymmetry at the Tevatron [4].

While measurements of the total cross section and differential distributions near their peaks are interesting, the collider center-of-mass energies at LHC Run-I (7,8 TeV) and Run-II (13 TeV) are sufficient to explore a qualitatively different region of phase space, where the top quarks are produced with energies much larger than their mass. From a theoretical perspective, such "boosted" top-quark production is quantified by the condition that top-pairs are produced in partonic collisions where $\hat{s} \gg m_{t}^{2}$. This regime is especially interesting phenomenologically, as it is sensitive to the possible new physics scale beyond the electroweak scale of the Standard Model. However, application of fixed-order perturbation theory in the boosted regime is problematic due to the appearance of potentially large corrections from soft and small-mass logarithms.

The purpose of this talk is to present a resummation formalism tailored for QCD calculations of differential cross sections in the boosted regime, and to briefly explore its implications on phenomenology. The results presented are taken from a detailed phenomenological analysis which is in progress [5]. The underlying theoretical basis for these results was set up in [6,7], and some higher-order perturbative results allowing precision applications were derived in $[8,9]$.

\section{Mellin-space resummation for (boosted) top production}

In this section we present the resummation formalisms for both the soft limit and the boosted soft limit. QCD factorization allows one to write the differential cross section with respect to the top-pair invariant mass $M$ and the scattering angle as

$$
\frac{d^{2} \sigma(\tau)}{d M d \cos \theta}=\frac{8 \pi \beta_{t}}{3 s M} \sum_{i j} \int_{\tau}^{1} \frac{d z}{z} \mathscr{L}_{i j}\left(\tau / z, \mu_{f}\right) C_{i j}\left(z, M, m_{t}, \cos \theta, \mu_{f}\right),
$$

where $z=M^{2} / \hat{s}, \tau=M^{2} / s, \beta_{t}=\sqrt{1-4 m_{t}^{2} / M^{2}}$, and $s$ is the hadronic center-of-mass energy squared. The perturbatively calculable hard-scattering kernels $C_{i j}$ are related to the partonic cross sections, with $i j$ denoting the partons in the initial state. The parton luminosities $\mathscr{L}_{i j}$ are nonperturbative functions defined as the convolution of parton distribution functions (PDFs). 
We shall consider the following two kinematic limits of the hard-scattering kernels:

$$
\begin{aligned}
\text { soft limit: } & \hat{s}, t_{1}, m_{t}^{2} \gg \hat{s}(1-z)^{2}, \\
\text { boosted soft limit: } & \hat{s}, t_{1} \gg m_{t}^{2} \gg \hat{s}(1-z)^{2} \gg m_{t}^{2}(1-z)^{2}
\end{aligned}
$$

where the definition of the Mandelstam variable $t_{1}$ is as in [6]. In each of these cases the perturbative expansions of the hard-scattering kernels contain large logarithms of scale ratios at each order in perturbation theory. In particular, the presence of a soft scale $\sqrt{\hat{s}}(1-z)$ introduces into the hardscattering kernel singular plus distributions of the form

$$
P_{n}(z)=\left[\frac{\ln ^{n}(1-z)}{1-z}\right]_{+},
$$

and the presence of a collinear scale $m_{t}$ in the boosted limit leads to large logarithms of the form $\ln ^{n}\left(m_{t}^{2} / \hat{s}\right)$. In the next two subsections, we discuss formalisms to resum these logarithms to all orders in perturbation theory.

For the discussion of resummation that follows, it is convenient to study the cross section in Mellin space. We define the Mellin transform and its inverse by

$$
\tilde{f}(N)=\mathscr{M}[f](N)=\int_{0}^{1} d x x^{N-1} f(x), \quad f(x)=\mathscr{M}^{-1}[\tilde{f}](x)=\frac{1}{2 \pi i} \int_{c-i \infty}^{c+i \infty} d N x^{-N} \tilde{f}(N),
$$

where the integration contour in the inverse transform is chosen such that it lies to the right of all singularities in the function $\tilde{f}(N)$. Convolutions such as the differential cross section in Eq. (2.1) become simple products in Mellin space. Indeed, the Mellin transform of Eq. (2.1) with respect to $\tau$ reads

$$
\frac{d^{2} \widetilde{\sigma}(N)}{d M d \cos \theta}=\frac{8 \pi \beta_{t}}{3 s M} \sum_{i j} \widetilde{\mathscr{L}_{i j}}\left(N, \mu_{f}\right) \widetilde{c}_{i j}\left(N, M, m_{t}, \cos \theta, \mu_{f}\right) .
$$

The limit $z \rightarrow 1$ corresponds to $N \rightarrow \infty$ in Mellin space, with plus distributions related to logarithms of $\bar{N} \equiv N e^{\gamma_{E}}$. In Mellin space, the soft and boosted soft limits in Eq. (2.2) and (2.3) are

$$
\begin{gathered}
\text { Mellin-space soft limit: } \quad \hat{s}, t_{1}, m_{t}^{2} \gg \frac{\hat{s}}{N^{2}}, \\
\text { Mellin-space boosted soft limit: } \quad \hat{s}, t_{1} \gg m_{t}^{2} \gg \frac{\hat{s}}{N^{2}} \gg \frac{m_{t}^{2}}{N^{2}} .
\end{gathered}
$$

We finally note that while Eq. (2.1) is written for the differential cross section with respect to the top-pair invariant mass $M$, in the soft limit one can easily perform a change of variable to obtain differential cross section with respect to the top quark transverse momentum $p_{T}$. Of course, contributions away from the soft limit are different in the two cases, but we can take them into account at fixed-order through a matching procedure to be discussed later.

\subsection{The soft limit}

In the soft limit, the resummed hard-scattering kernel can be written as 


$$
\begin{aligned}
\widetilde{c}_{i j}\left(N, M, m_{t}, \cos \theta, \mu_{f}\right)=\operatorname{Tr}\left[\widetilde{\boldsymbol{U}}_{i j}^{m}\left(\mu_{f}, \mu_{h}, \mu_{s}\right) \boldsymbol{H}_{i j}^{m}\left(M, m_{t}, \cos \theta, \mu_{h}\right) \widetilde{\boldsymbol{U}}_{i j}^{m \dagger}\left(\mu_{f}, \mu_{h}, \mu_{s}\right)\right. \\
\left.\quad \times \widetilde{\boldsymbol{s}}_{i j}^{m}\left(\ln \frac{M^{2}}{\bar{N}^{2} \mu_{s}^{2}}, M, m_{t}, \cos \theta, \mu_{s}\right)\right]+\mathscr{O}\left(\frac{1}{N}\right) .
\end{aligned}
$$

The formula consists of two main elements. Firstly, the hard functions $\boldsymbol{H}_{i j}^{m}$ and the soft functions $\widetilde{\boldsymbol{s}}_{i j}^{m}$ are matching functions which contain contributions from the two widely separated scales in (2.7). Secondly, the evolution factors $\widetilde{\boldsymbol{U}}_{i j}^{m}$ arise from solving the renormalization group (RG) equations of the hard and soft functions, and can be written as path-ordered exponentials of the anomalous dimensions governing their evolution. For details about these functions, we refer the readers to [10].

The philosophy of RG-improved perturbation theory is to choose the hard and soft scales around their natural values $\mu_{h} \sim M$ and $\mu_{s} \sim M / \bar{N}$, such that large logarithms are absent from the hard and soft functions. These large logarithms are exponentiated into the RG evolution factors, and the resummation of them is thus achieved. The logarithmic orders are counted by treating the large logarithms such as $\ln \left(\mu_{h} / \mu_{s}\right)$ as $\mathscr{O}\left(1 / \alpha_{s}\right)$. For next-to-next-to-leading logarithmic (NNLL) accuracy, we need the hard and soft functions to 1-loop, the various anomalous dimensions to 2-loop, and the 3-loop cusp anomalous dimension, which were calculated or collected in [10].

\subsection{The boosted soft limit}

The boosted soft limit (2.3) can be regarded as the $m_{t} \rightarrow 0$ limit of the soft limit. Taking $m_{t} \rightarrow 0$ in the resummation formula (2.9), one sees that both the hard and soft functions develop collinear logarithms of the form $\ln ^{n}\left(m_{t} / M\right)$. It has been demonstrated that the hard and soft functions can be further factorized in this limit as [6]

$$
\begin{aligned}
\boldsymbol{H}_{i j}^{m}\left(M, m_{t}, \cos \theta, \mu_{f}\right) & =\boldsymbol{H}_{i j}\left(M, \cos \theta, \mu_{f}\right) C_{D}^{2}\left(m_{t}, \mu_{f}\right)+\mathscr{O}\left(\frac{m_{t}}{M}\right), \\
\widetilde{\boldsymbol{s}}_{i j}^{m}\left(\ln \frac{M^{2}}{\bar{N}^{2} \mu_{f}^{2}}, M, m_{t}, \cos \theta, \mu_{f}\right) & =\widetilde{\boldsymbol{s}}_{i j}\left(\ln \frac{M^{2}}{\bar{N}^{2} \mu_{f}^{2}}, M, \cos \theta, \mu_{f}\right) \widetilde{s}_{D}^{2}\left(\ln \frac{m_{t}}{\bar{N} \mu_{f}}, \mu_{f}\right)+\mathscr{O}\left(\frac{m_{t}}{M}\right) .
\end{aligned}
$$

In the above formulas, the hard functions $\boldsymbol{H}_{i j}$ and soft functions $\widetilde{\boldsymbol{s}}_{i j}$ without the superscript $m$ are independent of the top quark mass $m_{t}$. They were calculated to NNLO in [8] and [9], respectively. All the $m_{t}$-dependence is now factorized into the two functions $C_{D}$ and $\widetilde{s}_{D}$, which are related to the perturbative heavy-quark fragmentation function, and were extracted at NNLO in [6]. Again solving RG equations for the component functions, the result for the resummed hard scattering kernel in the boosted soft limit is ${ }^{1}$

$$
\begin{aligned}
\widetilde{c}_{i j}\left(N, M, m_{t}, \cos \theta, \mu_{f}\right)=\operatorname{Tr}\left[\widetilde{\boldsymbol{U}}_{i j}\left(\mu_{f}, \mu_{h}, \mu_{s}\right) \boldsymbol{H}_{i j}\left(M, \cos \theta, \mu_{h}\right) \widetilde{\boldsymbol{U}}_{i j}^{\dagger}\left(\mu_{f}, \mu_{h}, \mu_{s}\right)\right. \\
\left.\quad \times \widetilde{\boldsymbol{s}}_{i j}\left(\ln \frac{M^{2}}{\bar{N}^{2} \mu_{s}^{2}}, M, \cos \theta, \mu_{s}\right)\right] \widetilde{U}_{D}^{2}\left(\mu_{f}, \mu_{d h}, \mu_{d s}\right) C_{D}^{2}\left(m_{t}, \mu_{d h}\right) \widetilde{s}_{D}^{2}\left(\ln \frac{m_{t}}{\bar{N} \mu_{d s}}, \mu_{d s}\right)
\end{aligned}
$$

\footnotetext{
${ }^{1}$ In the presence of heavy-quark loops the factorization of the partonic cross sections is more involved, therefore, we add such contributions onto the resummation formula (2.12) using fixed-order perturbation theory.
} 


$$
+\mathscr{O}\left(\frac{1}{N}\right)+\mathscr{O}\left(\frac{m_{t}}{M}\right) .
$$

The evolution factors $\widetilde{\boldsymbol{U}}_{i j}$ and $\widetilde{U}_{D}$ resum large logarithms when the matching scales are chosen to be near their canonical values: $\mu_{h} \sim M, \mu_{s} \sim M / \bar{N}, \mu_{d h} \sim m_{t}, \mu_{d s} \sim m_{t} / \bar{N}$. This resummation can be performed at NNLL accuracy as in the soft case discussed earlier. However, since the component functions in the boosted soft limit are known to NNLO in perturbation theory, we can also incorporate them into the resummation formula. We follow common nomenclature and refer to this accuracy as NNLL', where the ' emphasizes that the matching functions are known to one order higher than needed in a pure NNLL calculation (such as that in the soft limit described above).

\section{Matching across kinematic limits}

The resummations derived above are based on factorization formulas applicable in the soft or boosted soft limit. These factorization formulas are only valid up to leading order in their relevant expansion parameters, so every time a resummation is performed some information on subleading corrections is thrown away. However, these subleading corrections can be taken into account through a matching procedure. In our case, the optimal combination of the resummed formulas in the boosted soft and soft limit can be combined with fixed-order calculations according to

$$
d \sigma^{\mathrm{NLO}+\mathrm{NNLL}^{\prime}}=d \sigma^{\mathrm{NNLL}^{\prime b}}+\left(d \sigma^{\mathrm{NNLL}^{m}}-\left.d \sigma^{\mathrm{NNLL}^{b}}\right|_{\substack{\mu_{\mathrm{d}}=\mu_{s} \\ \mu_{\mathrm{dh}=\mu_{h}}}}\right)+\left(d \sigma^{\mathrm{NLO}}-\left.d \sigma^{\mathrm{NNLL}}\right|_{\substack{\mu_{\mathrm{s}}=\mu_{f} \\ \mu_{\mathrm{h}=\mu_{f}}}}\right) .
$$

The logarithmic counting has been defined above, and the superscripts $b$ and $m$ refer to results valid in the boosted soft and soft limits respectively. The first term in the above equation is the NNLL' resummation formula in the boosted soft limit. The difference of terms in the first bracket takes into account corrections from NNLL resummation in the soft limit which vanish in the limit $m_{t} \rightarrow 0$ and are therefore not contained in the first term. Finally, the difference of terms in the second bracket takes into account corrections from fixed-order perturbation theory which are subleading in the $z \rightarrow 1$ limit and thus accounted for by neither of the first two terms. It is straightforward to extend the above procedure to incorporate the recent NNLO calculations in [2]; however, we prefer to do this only once predictions with the dynamical scale choices used in our numerical calculations in the next section (i.e. not $\mu_{f}=m_{t}$ ) are available.

\section{Phenomenology}

We now provide numerical results derived from the resummation formulas described above. These results are obtained by first evaluating the Mellin transformed cross section (2.6) at a given point in phase space, and then performing the inverse Mellin transform numerically using the Minimal Prescription [11]. This procedure requires an efficient construction of Mellin-transformed parton luminosities: for this we use methods described in $[12,13]$.

In all our numerics we choose $m_{t}=173.2 \mathrm{GeV}$ and use MSTW2008NNLO PDFs [14]. The default matching scales are fixed at their canonical values listed above. The factorization scale is 


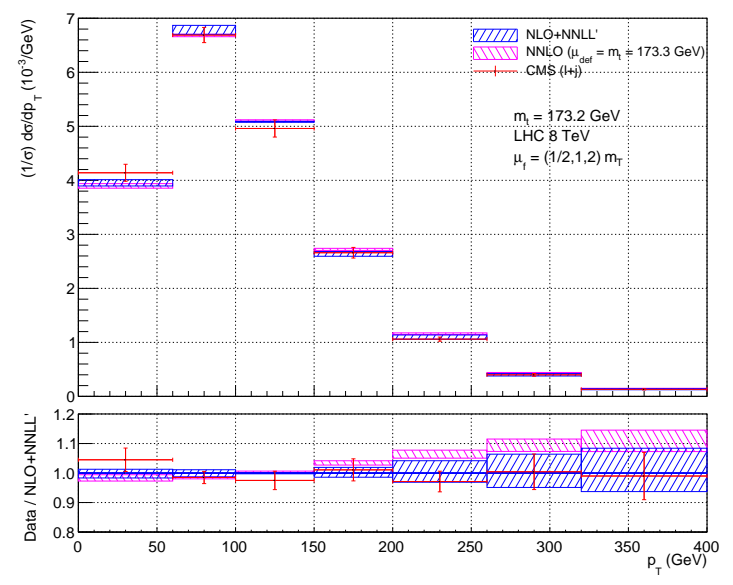

(a)

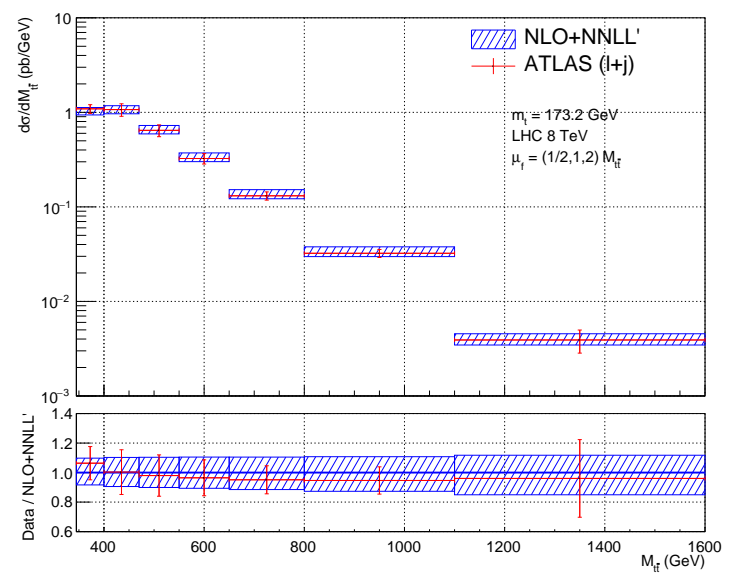

(c)

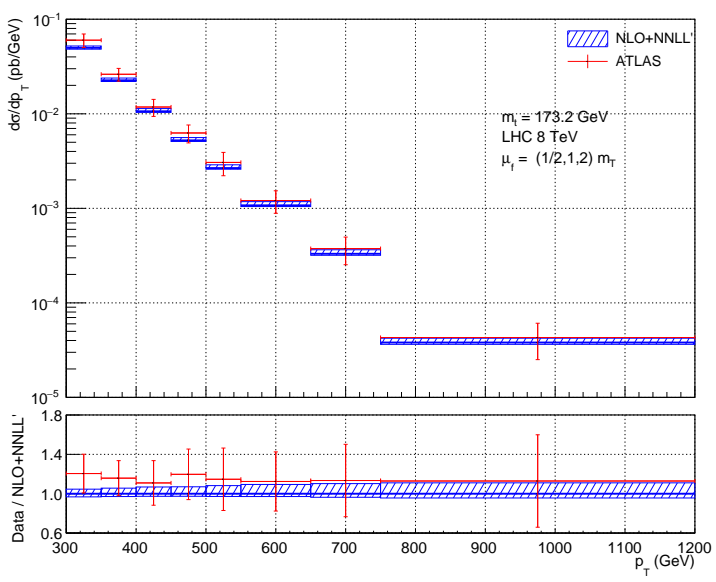

(b)

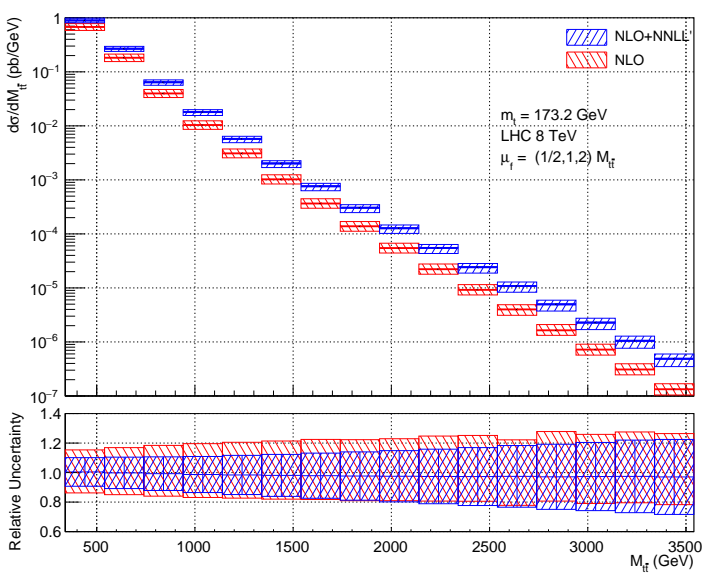

(d)

Figure 1: Numerical results as explained in the text. Note that while the bottom panels of (a)-(c) display ratios of predictions, that in (d) displays relative uncertainties.

correlated with the observables under consideration: for the invariant mass distribution its default value is $\mu_{f}=M$, while for the transverse momentum distribution it is set equal to the transverse mass $\mu_{f}=m_{T} \equiv \sqrt{p_{T}^{2}+m_{t}^{2}}$. The scale uncertainties are estimated by varying all scales independently by factors of two around their default values and combining the resulting variations in quadrature. The results of our analysis are shown in Figure 1.

In Figure 1 (a), we show our NLO+NNLL' prediction for the top-quark transverse momentum distribution in the relatively low energy (unboosted) region, compared with a recent experimental measurement in the lepton+jet channel by the CMS collaboration [15] and also the NNLO results from [2]. ${ }^{2}$ One sees that the NLO+NNLL' prediction agrees very well with the data, producing a slightly softer distribution at higher- $p_{T}$ than the NNLO calculation.

In Figure 1 (b), we present the top-quark transverse momentum distribution in the high- $p_{T}$

\footnotetext{
${ }^{2}$ We note that in [2], fixed scales with default values $\mu_{r}=\mu_{f}=m_{t}$ have been used, and a slightly different top quark mass $m_{t}=173.3 \mathrm{GeV}$ has been chosen.
} 
(boosted) region, where both the soft and the small-mass logarithms resummed through our formalism are expected to be relevant. We compare our results with a recent experimental measurement by the ATLAS collaboration [16] carried out by using fat-jet techniques especially designed for boosted kinematics. We find that our prediction agrees well with the data, although the experimental uncertainties are rather large.

In Figure 1 (c), we compare the top-pair invariant mass distribution with measurements from the ATLAS collaboration [16]. The two are in good agreement, although especially in the highest invariant-mass bin the experimental uncertainties are significant.

Finally, in Figure 1 (d), we display results reaching up to very high top-pair invariant mass. The results show significant resummation effects compared to NLO (where $\mu_{f}=M$ by default), although as seen in the bottom panel of that plot the relative uncertainties are roughly equal.

Run-II of the LHC will produce much more data in the boosted regime, which will allow for higher-precision comparisons of predictions and data for differential distributions. It will also be interesting to compare (and above all, match) with the NNLO results once they are available in the high- $p_{T}$ region with dynamical scale settings.

\section{References}

[1] V. del Duca and E. Laenen, arXiv:1510.06690 [hep-ph].

[2] M. Czakon, D. Heymes and A. Mitov, arXiv:1511.00549 [hep-ph].

[3] M. Czakon, P. Fiedler and A. Mitov, Phys. Rev. Lett. 110, 252004 (2013) [arXiv:1303.6254 [hep-ph]].

[4] M. Czakon, P. Fiedler and A. Mitov, Phys. Rev. Lett. 115, 052001 (2015) [arXiv:1411.3007 [hep-ph]].

[5] A. Ferroglia, B. D. Pecjak, D. J. Scott, X. Wang and L. L. Yang, in preparation.

[6] A. Ferroglia, B. D. Pecjak and L. L. Yang, Phys. Rev. D 86, 034010 (2012) [arXiv:1205.3662 [hep-ph]].

[7] A. Ferroglia, S. Marzani, B. D. Pecjak and L. L. Yang, JHEP 1401, 028 (2014) [arXiv:1310.3836 [hep-ph], arXiv:1310.3836].

[8] A. Broggio, A. Ferroglia, B. D. Pecjak and Z. Zhang, JHEP 1412, 005 (2014) [arXiv:1409.5294 [hep-ph]].

[9] A. Ferroglia, B. D. Pecjak, L. L. Yang, B. D. Pecjak and L. L. Yang, JHEP 1210, 180 (2012) [arXiv:1207.4798 [hep-ph]].

[10] V. Ahrens, A. Ferroglia, M. Neubert, B. D. Pecjak and L. L. Yang, JHEP 1009097 (2010) [arXiv:1003.5827 [hep-ph]].

[11] S. Catani, M. L. Mangano, P. Nason and L. Trentadue, Nucl. Phys. B 478, 273 (1996) [hep-ph/9604351].

[12] M. Bonvini and S. Marzani, JHEP 1409, 007 (2014) [arXiv:1405.3654 [hep-ph]].

[13] M. Bonvini, arXiv:1212.0480 [hep-ph].

[14] A. D. Martin, W. J. Stirling, R. S. Thorne and G. Watt, Eur. Phys. J. C 63, 189 (2009) [arXiv:0901.0002 [hep-ph]].

[15] V. Khachatryan et al. [CMS Collaboration], Eur. Phys. J. C 75, 542 (2015) [arXiv:1505.04480 [hep-ex]]. 
[16] G. Aad et al. [ATLAS Collaboration], arXiv:1510.03818 [hep-ex].

[17] G. Aad et al. [ATLAS Collaboration], arXiv:1511.04716 [hep-ex]. 